\title{
Quaternary Ammonium Polyethylenimine Nanoparticles
}

National Cancer Institute

\section{Source}

National Cancer Institute. Quaternary Ammonium Polyethylenimine Nanoparticles. NCl

Thesaurus. Code C88305.

A crosslinked nanoparticle formulation containing quaternary ammonium

polyethylenimine (QA-PEI) with potential antibacterial activity. The cationic polymer PEI kills bacteria by rupturing their cell membranes without the development of resistance. Quaternary ammonium polyethylenimine nanoparticles can be incorporated into dental composite resins or silicon obturator prostheses and may prevent or delay bacterial growth. 